BNL-73471-2005-CP

\title{
IR Optics Measurement with Linear Coupling's Action-Angle Parameterization
}

\author{
Y. Luo, M. Bai, F. Pilat, T. Satogata, D. Trbojevic \\ Presented at the Particle Accelerator Conference (PAC'05) \\ Knoxville, Tennessee \\ May 16-20, 2005
}

Collider-Accelerator Department

Brookhaven National Laboratory

P.O. Box 5000

Upton, NY 11973-5000

www.bnl.gov

Managed by

Brookhaven Science Associates, LLC

for the United States Department of Energy under

Contract No. DE-AC02-98CH10886

This is a preprint of a paper intended for publication in a journal or proceedings. Since changes may be made before publication, this preprint is made available with the understanding that it will not be cited or reproduced without the permission of the author. 


\section{DISCLAIMER}

This report was prepared as an account of work sponsored by an agency of the United States Government. Neither the United States Government nor any agency thereof, nor any of their employees, nor any of their contractors, subcontractors, or their employees, makes any warranty, express or implied, or assumes any legal liability or responsibility for the accuracy, completeness, or any third party's use or the results of such use of any information, apparatus, product, or process disclosed, or represents that its use would not infringe privately owned rights. Reference herein to any specific commercial product, process, or service by trade name, trademark, manufacturer, or otherwise, does not necessarily constitute or imply its endorsement, recommendation, or favoring by the United States Government or any agency thereof or its contractors or subcontractors. The views and opinions of authors expressed herein do not necessarily state or reflect those of the United States Government or any agency thereof.

FOR UNCLASSLFIED, UNLIMITED STIPRODUCTS

Available electronically at:

OSTL:

http://www.osti.gov/bridge

Available for a processing fee to U.S. Department of Energy and its contractors, in paper from:

U.S. Department of Energy

Office of Scientific and Technical Information

P.O. Box 62

Oak Ridge, TN 37831

Phone: (865) 576-8401

Facsimile: (865) 576-5728

E-mail: reports@adonis.osti.gov

National Technical Information Service (NTIS):

Availablefor sale to the public from:

U.S. Department of Commerce

National Technical Information Service

5285 Port Royal Road

Springfield, VA 22131

Phone: (800) 553-6847

Facsimile: (703) 605-6900

Online ordering: http://www.ntis.gov/ordering.htm

Q Fristad an retyelad pangr 


\title{
IR optics Measurement with linear coupling's action-angle parameterization *
}

\author{
Y. Luo, M. Bai, F. Pilat, T. Satogata, D. Trbojevic \\ Brookhaven National Laboratory, Upton, NY 11973, USA
}

\section{Abstract}

A parameterization of linear coupling in action-anglecoordinates is convenient for analytical calculations and interpretation of turn-by-turn(TBT) beam position monitor (BPM) data. We demonstrate how to use this parameterization to extract the twiss and coupling parametersin interaction regions (IRs), using BPMs on each side of the long IR driftregion. The example of TBTBPM analysis was acquired at the Relativistic Heavy Ion Collider(RHIC), using an $\mathrm{AC}$ dipole to excite a single eigenmode. Besides the full treatment, a fast estimate of beta*, the beta function at the interaction point (IP), is provided, along with the phase advance between these BPMs. We also calculate and measure the waist of the beta function and the local optics.

\section{BACKGROUND}

\section{Action-angle parameterization}

For general two-dimensionallinearly coupled motion, a single particle's motion is representedas [1]

$$
\left(\begin{array}{c}
x \\
x^{\prime} \\
y \\
y^{\prime}
\end{array}\right)=\mathbf{P}\left(\begin{array}{c}
\sqrt{2 J_{I}} \cos \Phi_{I} \\
-\sqrt{2 J_{I}} \sin \Phi_{I} \\
\sqrt{2 J_{I I}} \cos \Phi_{I I} \\
-\sqrt{2 J_{I I}} \sin \Phi_{I I}
\end{array}\right),
$$

where $J_{I, I I}$ are the globally constant actions of the two eigenmodes. $\Phi_{I, I I}$ are the eigenmode phases.

$\mathbf{P}$ is the transfer matrix from the laboratory coordinate system to the action-angle coordinate system. It can be analytically calculated from the eigenvectors of the oneturn $4 \times 4$ transfer matrix. $\mathrm{P}$ can also be defined in terms of Twiss and coupling parameters given by the EdwardsTeng's parameterization. This definition can be inverted to provide Twiss and coupling parameters in terms of $\mathbf{P}$. For example, for eigen mode $\mathrm{l}$,

$$
\begin{gathered}
\left\{\begin{array}{l}
\beta_{I}=\frac{p_{1 I}}{p_{22}} \\
\alpha_{I}=-\frac{p_{21}}{p_{22}} \\
\gamma_{I}=\frac{1+\alpha_{I}^{2}}{\beta_{I}}=\frac{p_{21}^{2}+p_{22}^{2}}{p_{11} p_{22}}
\end{array}\right. \\
r=\sqrt{p_{11} p_{22}}=\sqrt{p_{33} p_{44}} \\
\mathbf{C}=r \mathbf{P}_{12} \mathbf{P}_{22}^{-1}=-\frac{1-r^{2}}{r} \mathbf{P}_{11} \mathbf{P}_{21}^{-1}
\end{gathered}
$$

* Work supported by U.S. DOE under contract No DE-AC0298CH10886

\section{Excitation of one eigenmode}

To measure Twiss parameters, a single eigenmodemotion is excited [2, 3]. In the RHIC, $\mathbf{A C}$ dipoles are used for this purpose [4, 5]. For simplicity, Eq. (1) can be rewritten as

$$
\begin{gathered}
\left(\begin{array}{c}
x \\
x^{\prime} \\
y \\
y^{\prime}
\end{array}\right)=\mathbf{F}\left(\begin{array}{c}
\cos \Phi_{I} \\
-\sin \Phi_{I} \\
\cos \Phi_{I I} \\
-\sin \Phi_{I I}
\end{array}\right), \\
\mathbf{F}=\left(\begin{array}{cccc}
\mathrm{P} 11 \sqrt{J_{I}} & 0 & p_{13} \sqrt{J_{I I}} & p_{14} \sqrt{J_{I I}} \\
p_{21} \sqrt{J_{I}} & \mathrm{P} 22 \sqrt{J_{I}} & p_{23} \sqrt{J_{I I}} & p_{24} \sqrt{J_{I I}} \\
\mathrm{P} 31 \sqrt{J_{I}} & p_{32} \sqrt{J_{I}} & \mathrm{P} 33 \sqrt{J_{I I}} & 0 \\
p_{41} \sqrt{J_{I}} & p_{42} \sqrt{J_{I}} & \mathrm{P} 43 \sqrt{J_{I I}} & p_{44} \sqrt{J_{I I}}
\end{array}\right)
\end{gathered}
$$

where $\mathbf{F}$ includes the action information.

In this article, we assume that eigenmode I is more closely related to the horizontal plane than is eigenmode 11 , whilst eigenmodeII is more associated with the vertical plane than eigenmodeI. Thus, if only eigenmode I is activated, the elements in the last two columns of $\mathbf{F}$ are zero, If only eigenmode 11 is activated, the elements in the first two columns of $\mathbf{F}$ are zero. The matrix $\mathbf{F}$ can be obtained from TBT BPM data at a single ring BPM

\section{TBT data at DX BPMs}

In each $\mathbb{R}$ of the RHIC, there are two dual-planeBPMs that are close to the IR's DX separation magnets and face to the interaction point. TheseBPMs are called DX BPMs. There is no othermagnetbetween the two DX BPMs in the IR if we ignore the effect of the detector magnet. Therefore, the TBT angles $\left(x^{\prime}, y^{\prime}\right)$ at the two DX BPMs can be determined,

$$
\left\{\begin{array}{l}
x^{\prime}=\frac{x_{2}-x_{1}}{2 L_{2}} \\
y^{\prime}=\frac{y_{2}-y_{1}}{2 L}
\end{array} .\right.
$$

$2 L$ is the distance between the two DX BPMs; for RHIC, $2 \mathrm{~L}=16.652 \mathrm{~m} .\left(x_{1}, y_{1}\right)$ are the BPM position readings at the up-stream DX BPM, $\left(x_{2}, y_{2}\right)$ are the BPM position readings at the down-stream DX BPM. These angles are constant across the $\mathbb{R}$ drift.

\section{Propagations of opticsparameters in the IR drift}

Propagation of the Twiss and coupling parameters through the IR drift is used for determination $O$ the beta waist and to provide a fast estimate of $\beta^{*}$. At the $\beta$ waist, 
where $\alpha_{I, I I}=0$,

$$
\mathbf{P}_{w}=\left(\begin{array}{cccc}
p_{11} & 0 & p_{13} & p_{14} \\
0 & \mathbf{P 2 2} & \mathbf{P 2 3} & \mathbf{P 2 4} \\
\mathbf{P 3 1} & \mathbf{P 3 2} & \mathbf{P 3 3} & \mathbf{0} \\
\mathbf{P 4 1} & \mathbf{P 4 2} & \mathbf{0} & p_{44}
\end{array}\right)
$$

For simplicity, we assumed that eigenmode I and eigenmode II's $\beta$ waists are located at the same point. Even if they are not, the following conclusions still hold. $\mathbf{T}_{1 \rightarrow 2}$ is the $\mathbf{4} \times \mathbf{4}$ drift transfer matrix from the waist to a point in the $\mathbb{R}$ diff, say point $2 . l$ is the distance from the waist to point 2. Matrix $\mathbf{G}$ defined by [1]'s Eq. (69) is

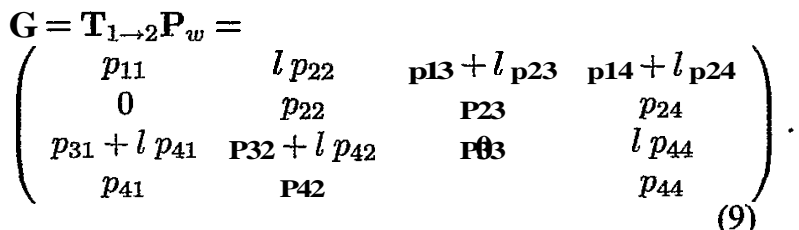

According to Eqs. (78) and (79) in [1], and considering Eq.(4) at the waist, the Twiss parameters at point 2 can be determined with the above $\mathbf{G}$,

$$
\left\{\begin{array}{l}
\tilde{\beta}_{I}=\frac{p_{1,}^{2}+l^{2} p_{22}}{p_{1,1} p_{22}}-\beta_{w, I}+\frac{l^{2}}{\beta_{w, I}} \\
\alpha_{I}=-\frac{l p_{22}^{2}}{p_{11} p_{22}}=-\frac{l}{\beta_{w, I}}
\end{array} .\right.
$$

The subscript $\mathrm{w}$ means the parameters are those at the $\beta$ waist.

Therefore, knowing the Twiss parameters at point 2 , the location of the $\beta$ waist and its $\beta$ value canbe calculated,

$$
\begin{gathered}
\beta_{w, I}=\frac{\widetilde{\beta}_{I}}{1+\widetilde{\alpha}_{r}^{2}}, \\
\delta l_{w, I}=-\widetilde{\alpha}_{I} \beta_{w, I} .
\end{gathered}
$$

$\delta l_{w, I}$ is the longitudinal locations of eigenmodeI's waists with respect to point 2.

According to Eqs. (70) and (72) in [1], the phase advance of the eigenmode $I$ from the $\beta$ waist to point 2 are given by $\mathbf{G}$,

$$
\Delta \Phi_{I}=\tan ^{-1}\left(\frac{l p_{92}}{p_{11}}\right)=\tan ^{-1}\left(\frac{1}{\beta_{w, I}}\right)
$$

Assuming that the beta waist is located in the middle of the two DX BPMs, the total phase advance between them is

$$
\Delta \Phi_{I}=2 \tan ^{-1}\left(\frac{L}{\beta_{w, I}}\right),
$$

where $\mathrm{L}$ is the distance from the IR center to the DX BPM. Eq. (14) can be used for a fast estimate of the $\beta_{c}$ at the IR's center,

$$
\beta_{c, I}=\frac{L}{\tan \frac{\Delta \Phi_{I}}{2}}
$$

One particular advantage of this estimate is that it relies only on BPM TBT phase advance measurements, which are insensitive to BPM gain and offset errors.

Further, knowing the coupling matrix $\mathbf{C}$ at one point in the IR drift, the coupling matrix $\widetilde{\mathrm{C}}_{w}$ at the $\beta$ waist in the $\mathbb{R}$ drift can be obtained. According Eq.(86) in [1],

$$
\begin{aligned}
\mathbf{C}_{w} & =\mathbf{M}_{d} \mathbf{C M}_{d}^{-1} \\
\mathbf{M}_{d} & =\left(\begin{array}{cc}
1 & \delta l_{w} \\
0 & 0
\end{array}\right),
\end{aligned}
$$

where 61 , is the longitudinal drift length from point 2 to the waist.

A similar calculation gives the propagation of eigenmode II.

\section{MEASUREMENTS}

In the following, an example is given demonstratinghow to use the linear coupling's action-angleparameterization to extract the optics parameters in the IR. BPM data used here was acquired with an $\mathrm{AC}$ dipole excitation at eigenmode I's fractional tune frequency

To clarify the data processing procedure, we first calculate the Twiss and coupling parameters at the Blue ring IR6 center. The two dual-planeDX BPMs are rbpm.b-g5 and rbpm.b-g6. The Blue beam circulates from rbpm.b-g5 to rbpm.b-56. The TBT position data at the IR center are given by

$$
\left\{\begin{array}{l}
x_{c}=\frac{x_{1}+x_{2}}{2} \\
y_{c}=\frac{y_{1}+y_{2}}{2}
\end{array} .\right.
$$

\section{Matrix F}

With the TBT $\left(x_{c}, x_{c}^{\prime}, y_{c}, y_{c}^{\prime}\right)$ data at Blue ring IR G center, $\mathbf{F}$ is calculated with harmonic analysis,

$$
\mathbf{F}=\left(\begin{array}{cccc}
1.244 \times 10^{-5} & 0 . & 0 & 0 \\
-1.009 \times 10^{-6} & 1.273 \times 10^{-5} & 0 & 0 \\
-6.491 \times 10^{-7} & -9.74470^{-7} & 0 & 0 \\
-4.509 \times 10^{-7} & -9.293 \times 10^{-7} & 0 & 0
\end{array}\right)
$$

In this article, the SI unit system is used.

$$
\boldsymbol{r} \text { and } \sqrt{J_{I}}
$$

At $J R G$ center,

$$
r \sqrt{J_{I}}=\sqrt{F_{11} F_{22}}=1.2583 \times 10^{-5}\left[(\mathrm{~m} . \mathrm{rad})^{-\frac{1}{2}}\right] .
$$

and

$$
\left\|\mathbf{F}_{21}\right\|=\frac{\left\|\mathbf{P}_{a 1}\right\|}{\left\|\mathbf{P}_{11}\right\|}=\frac{1-r^{2}}{r^{2}}-0.001035
$$

Therefore,

$$
\begin{gathered}
r=0.9995 \\
\sqrt{J_{I}}=1.259 \times 10^{-5}(\mathrm{~m} . \mathrm{rad})^{-\frac{1}{2}} .
\end{gathered}
$$

When $r$ is close to 1 , the optics is well decoupled locally at that point. $J_{I}$ is a global constant. 


\section{Twiss and couplingparameters}

According Eq.(2), from Eq. (19), the eigenmode I's Twiss parameters at the IR6 center are

$$
\begin{gathered}
\beta_{c, I}=\frac{p_{11}}{p_{22}}=\frac{F_{11}}{F_{22}}=0.9771 \mathrm{~m} \\
\alpha_{c, I}=-\frac{p_{21}}{p_{22}}=-\frac{F_{21}}{F_{22}}-0.0793
\end{gathered}
$$

According to Eqs.(4) and (6), the coupling matrix at the IR6 center can be calculated through $\mathbf{F}$,

$$
\mathbf{C}_{c}=\left(\begin{array}{cc}
0.0730 & -0.0765 \\
-0.0422 & 0.0584
\end{array}\right)
$$

\section{$\beta_{Y}$ waist determination}

Knowing the eigenmode I's Twiss parameters at tne $\mathbb{R}$ center, accordingto Eq. (11), the IR6 $\beta$ waist can be determined,

$$
\begin{gathered}
\beta_{w, I}=0.9710 \mathbf{m} \\
\delta l_{w, I}=-0.0770 \mathrm{~m} .
\end{gathered}
$$

A negative $\delta l_{w, I}$ signifies the $\beta_{I}$ waist is sited upstream with respect to the IR center.

According to Eq. (16), the coupling matrix $\mathbf{C}_{w, I}$ at the eigenmode I's $\beta$ waist can be calculated from that at the center,

$$
\mathbf{C}_{w, I}=\left(\begin{array}{cc}
0.0763 & -0.0752 \\
-0.0422 & 0.0551
\end{array}\right)
$$

\section{Summary of IR optics parameters}

Table 1 lists all IR centers' $\beta_{c, I}$ s from the phase advances between the two adjacent DX BPMs, according to Eq. (15) . The phase advances between the relevant two DX BPMs also are given. The unit of the phase advances are given in unit of degree.

Table 2 lists the Twiss and coupling parameters at the $\mathbb{R}$ centers from the action-angle parameterization. Since the rbpm.b-g2 BPM vertical data is aberrant, the coupling parameters are not available at IR2 center.

Comparingthe $\beta_{c, I}$ s at the IR centers from Tables 1 and 2 , a big difference in the $\mathbb{R} 12$ is apparent. Looking into the turn-by-turn BPM data of the IR12 DX BPMs, I found that the downstream BPMrbpm.b-g12 gave poor horizontal data.

Table 3 lists the locations of $\beta_{w, I}$ waist and its $\beta_{w, I}$, coupling parameters there. $\delta l_{w, I}$ is the longitudinal offset with respect to the $\mathbb{R}$ center.

Table 4 lists the coupling parameter $\boldsymbol{r}$ and eigenmodeI's action $\sqrt{J_{I}}$ in IRs. $\sqrt{J_{I}}$ is a global constant. The average of $\sqrt{J_{I}}$ in the IRs in Table 4 is

$$
\overline{\sqrt{J_{I}}}=1.18 \times 10^{-5}(\mathrm{~m} \cdot \mathrm{rad})^{-\frac{1}{2}},
$$

\begin{tabular}{|l|c|c|c|c|c|c|}
\hline IRs & IR6 & IR8 & IR10 & IR12 & IR2 & IR4 \\
\hline$\Delta \Phi_{I}$ & 166.695 & 166.474 & 147.064 & 141.788 & 150.637 & 119.395 \\
\hline$\beta_{c, I}$ & 0.9711 & 0.9874 & 2.4613 & 2.8843 & 2.1815 & 4.8660 \\
\hline
\end{tabular}

Table 2: Twiss and coupling parameters at the IR centers from the linear coupling's action-andeparametrization

\begin{tabular}{|l|c|c|c|c|c|c|}
\hline Rs & $\beta_{c, I}$ & $\alpha_{c, I}$ & $c_{11}$ & $c_{12}$ & $c_{21}$ & $c_{22}$ \\
\hline IR6 & 0.9771 & 0.0793 & 0.0730 & -0.0765 & -0.0422 & 0.0584 \\
\hline IR8 & 0.9885 & 0.0335 & 0.2549 & -1.7128 & -0.0455 & 1.0318 \\
\hline IR10 & 2.4678 & 0.0536 & -0.0192 & -0.4945 & 0.0091 & -0.0213 \\
\hline IR12 & $\mathbf{3 . 2 7 6 2}$ & -0.3929 & 0.0484 & -1.2168 & $\mathbf{0 . 0 3 3 7}$ & -0.2773 \\
\hline IR2 & 2.5006 & 0.3972 & & & & \\
\hline IR4 & 4.8918 & -0.0844 & -0.0854 & $\mathbf{0 . 0 3 8 2 9}$ & $-\mathbf{0 . 0 3 4 4}$ & $-\mathbf{0 . 2 5 5 7}$ \\
\hline
\end{tabular}

Table 3: Optics parameters at the $\beta_{I}$ waist in IRs.

\begin{tabular}{|l|c|c|c|c|c|c|}
\hline IRs & $\delta l_{w, I}$ & $\beta_{w, I}$ & $c_{11}$ & $c_{12}$ & $c_{21}$ & $c_{22}$ \\
\hline IR6 & -0.0770 & 0.9710 & 0.0763 & -0.0752 & -0.0422 & 0.0551 \\
\hline IR8 & -0.0331 & 0.9874 & 0.2812 & -1.9039 & -0.0455 & 1.0318 \\
\hline IR10 & -0.1320 & 2.4607 & -0.0204 & -0.4955 & 0.0091 & -0.0213 \\
\hline IR4 & 0.4100 & 4.8571 & -0.1007 & -0.0261 & -0.0348 & -0.2445 \\
\hline
\end{tabular}

Table 4: $r$ and $\sqrt{J_{I}}$ in IRs.

\begin{tabular}{|l|c|c|}
\hline IRs & $r$ & $\sqrt{J_{Y}}\left[\mathrm{~m} . \mathrm{mrad}^{-\frac{1}{2}}\right.$ \\
\hline IR6 & 0.999 & $1.26 \times 10^{-5}$ \\
\hline IR8 & 0.911 & $1.17 \times 10^{-5}$ \\
\hline IR10 & 0.997 & $1.11 \times 10^{-5}$ \\
\hline RR4 & 0.988 & $1.09 \times 10^{-5}$ \\
\hline
\end{tabular}

which can be used to roughly extract the $r \sqrt{\beta_{I}}$ at the horizontal BPMs in the arcs,

$$
r \sqrt{\beta_{I}}=\frac{}{\sqrt{J_{I}}} .
$$

and $\frac{c_{12}}{r}$ at the dual-planeBPMs,

$$
\frac{c_{12}}{r}=\frac{F_{32}}{F_{11}} .
$$

\section{REFERENCES}

[1] Y. Luo, Phys. Rev. ST Accel. Beam 7,124001 (2004).

[2] D. Sagan, Phys. Rev. ST Accel. Beam 4, 122801 (2001).

[3] D. Sagan et al., Phys. Rev. ST Accel. Beam 3, 102801 (2000).

[4] M. Bai, et ah, "Measuring beta function and phase advance in RHIC with an AC dipole", in proceedings of the 2003 Particle Accelerator Conference,Portland, p2204.

[5] R. Calaga, et al., "RHICoptics measurements at different working point", in proceedings of the 2004 European Particle Accelerator Conference, Lucerne, Switzerland, p1541. 